\title{
Study on Regional Differences between Financial Agglomeration and Economic Growth
}

\author{
Liang Jinhua ${ }^{1,2}$ \\ ${ }^{1}$ Sichuan University, School of economics, Sichuan University, Chengdu, China \\ ${ }^{2}$ Zhejiang Gongshang University Hangzhou College of Commerce, Hangzhou, China
}

Keywords: financial risk; financial management; money growth; agglomeration; economic growth forecast

\begin{abstract}
In the paper, threshold of financial agglomeration and regional economic growth in Zhejiang Province from 2002 to 2016 was studied using threshold panel model. The test result indicates that the relationship between financial agglomeration and regional economic growth was not merely simple linear function relationship, but mingled with "double threshold effect". That is, when the financial agglomeration level was low, the increasing of financial agglomeration can inhibit economic growth; when the financial agglomeration level was in the medium range, the increasing of financial agglomeration can promote economic growth effective; and when the financial agglomeration level was relatively high, the increasing of financial agglomeration has adverse effect on economic growth. Hence, the writer puts forward strengthening coordination between financial industry and other industries, introducing financial talent, increasing financial resource turnover and improving infrastructure construction.
\end{abstract}

\section{Introduction}

In recent years, economic growth in Zhejiang is rapid, the per capita GDP of Zhejiang increases from 9,603 Yuan in 1998 to 63,472 Yuan in 2014, by nearly 7 times. With fast economic growth, financial agglomeration received widely attention. Undoubtedly, financial agglomeration played a core role in modern society, the corresponding external economic scale effect and diffusion effect promote the regional economic growth. In new economic node, the financial industry value accounts for more proportion in GDP and financial agglomeration exerts more significant impact on economic growth. As the subject of West Coast Economic Zone, it is necessary for Zhejiang Province makes effort to increase financial and economic level so as to suit the development requirement of new economic mode and explore the law between financial agglomeration and economic growth. Hence, the writer studies the relationship between the financial agglomeration and regional economic growth objectively with Zhejiang Province as research object, which has guidance significance on economic development direction and policy making of Zhejiang Province.

\section{Research Design}

Not only do panel data include cross section and period information, but also include 3D information of variable. Building panel modeling not only helps obtain more information, but also helps us to analyze relationship between variable and explained variable in depth. Threshold interval is divided according to data generation characteristics using "panel threshold model" developed by Hansen (1999). Further, the change relationship between economic situation in each region and the financial industrial agglomeration on different conditions is studied scientifically. Moreover, related suggestions about realizing steady and fast growth of regional economy of Zhejiang Province are proposed on such basis.

\section{Single threshold estimation method}

The single threshold model proposed by Hansen (1999) is set up as below: 


$$
y_{i t}=\beta^{\prime}{ }_{1} x_{i t} I\left(q_{i t}<\gamma\right)+\beta^{\prime}{ }_{2} x_{i t} I(q \geq \gamma)+e_{i t}+\mu_{i}
$$

In the equation, $(1 \leq i \leq n, 1 \leq t \leq T), \quad \mathrm{Y}_{\mathrm{it}}$ is the scalar composed of the explained variable, $\mathrm{x}_{\mathrm{it}}$ is the vector composed of the explained variable, $I$ is indicator function, $\beta_{12}$ is coefficient vector, $\mathrm{e}_{\mathrm{it}}$ is error term, $\mathrm{q}_{\mathrm{it}}$ is the scalar composed of threshold variable, and $\gamma$ is threshold value.

In equation (1), let $x i t(\gamma)=\left\{\begin{array}{l}x_{i t} I\left(q_{i t} \leq \gamma\right) \\ x_{i t} I\left(q_{i t} \geq \gamma\right)\end{array}\right.$, and $\beta=\left(\beta^{\prime}{ }_{1}, \beta^{\prime}{ }_{2}\right)$, then equation (1) is equivalent to:

$$
Y_{i t}=\mu_{i}+\beta^{\prime} x_{i t}(\gamma)+e_{i t}
$$

At this time, the observed samples are divided into two sections according to whether the threshold variable is smaller or more than threshold value $\gamma$. Regression coefficient in each section is $\left(\beta_{1}, \beta_{2}\right)$, and the explanatory variable $\mathrm{x}_{\mathrm{it}}$ in each section is variable. After removing a series of conversion like individual effect ${ }^{\mu_{\mathrm{i}}}$, equation (2) can be simplified as:

$$
Y=X(\gamma) \beta+e
$$

For this equation, $X(\gamma)=\left[x_{1}^{*}(\gamma), \ldots, x_{i}^{*}(\gamma), \ldots, x_{n}^{*}(\gamma)\right]^{-1}, \gamma$ is given at will, then coefficient value $\beta$ of each section can be obtained through using common least square estimate:

$$
\hat{\beta}(\gamma)=\left(X(\gamma)^{\prime} X(\gamma)\right)^{-1} X(\gamma)^{\prime} Y
$$

Thus, we get the residual error regression vector function as below:

$\hat{e}(\gamma)=Y-X(\gamma) \hat{\beta}(\gamma)$. Then, the sum function of squared errors is:

$$
S_{1}(\gamma)=\hat{e}(\gamma)^{\prime} \hat{e}(\gamma)=Y^{\prime}\left(I-X(\gamma)^{\prime}\left(X(\gamma)^{\prime} X(\gamma)\right)^{-1} X(\gamma)^{\prime}\right) Y
$$

After minimizing equation (5), the required estimated threshold value can be derived reversely, namely:

$$
\hat{\gamma}=\arg \min S_{1}(\gamma)
$$

After getting the estimated threshold value, the significance is tested. The original hypothesis is: $\mathrm{H}_{0} \square \beta_{1}=\beta_{2}$. Likelihood ration test statistics is:

$F_{1}=\left(S_{0}-S_{1}(\hat{\gamma})\right) / \hat{\sigma}^{2}$. Hereinto, $\beta_{1}$ is got through common least square regression, and $\mathrm{S}_{0}$ is the residual sum of squares on original hypothesis condition. When single threshold effect $\left(\beta_{1} \neq \beta_{2}\right)$ exists, the estimated $\hat{\gamma}$ is equal to $\gamma$, but the asymptotic distribution is off standard. Here, to test the truth of estimated value, the original hypothesis was built as:

$\mathrm{H}_{0} \square \gamma=\gamma_{0}$. Likelihood ration test statistics is:

$L R_{1}(\gamma)=\left(S_{1}(\gamma)-S_{1}(\gamma)\right) / \hat{\sigma}^{2}$. When $L R_{1}>-2 \log (1-\sqrt{1-\alpha})$, it rejects original hypothesis, and $\alpha$ is significant level.

The above narrated model method is used for single threshold model. The test method of multiple threshold is similar, so there is no unnecessary narration here. 


\section{Indicator selection}

The economic growth level is expressed with per capita GDP in this region and financial agglomeration level is weighed with location entropy of total loans and deposits of financial industry in each region.

The explained variable $\left(G D P_{i t}\right)$ : per capita GDP. The reason is that per capita GDP can reflect the economic growth level and difference in each region more accurately than regional GDP.

Threshold variable $\left(Q_{i t}\right)$ : financial agglomeration level. Here, location entropy is used to determine financial agglomeration level of Zhejiang Provinces. The formula is expressed as:

$$
Q_{i t}=\left(x_{i t} / q_{i t}\right) /\left(\Sigma_{(i)} x_{i t} / \Sigma_{i} \Sigma_{(t)} q_{i t}\right)
$$

Hereinto, $x_{i t}$ means total loans and deposits of $\mathrm{i}$ region in the $\mathrm{t}$ year, means population of $\mathrm{i}$ region in the $\mathrm{t}$ year, and $\Sigma_{(i)} x_{i t}$ means total financial deposits and loans in Zhejiang Province, and means total population in the t year. The greater $Q_{i t}$ is, it means the financial agglomeration level in this region is higher.

Control variable: to measure the effect relationship between financial agglomeration and regional economic growth accurately, fixed asset investment, consumption level, and industrial development level and foreign opening degree of region as the control variables.

Fixed asset investment $\left(\operatorname{Inv} v_{i t}\right)$ is expressed with per capita fixed asset investment. Research of Pu Xiaosong and Chen Wei (2009), Song Lizhi (2011) et al has proved that an influencing factor of regional economic growth is fixed asset investment. Here, per capita fixed asset investment is taken as a control variable, which conforms to the selection principle of control variable.

Consumption level $\left(\mathrm{Con}_{i t}\right)_{\text {is }}$ measured with per capital regional consumption amount. It is because consumption is the power of regional economic growth and the increasing of per capita consumption drives the regional economic growth.

Foreign opening degree $\left(\right.$ Fore $\left._{i t}\right)$ brings about many benefits to a region. For example, it produces more technological learning chances through importing, or introducing some advanced equipment. In addition, under the pressure of fierce international competition, it is urgent to increase foreign trade so as to elevate the regional competitiveness. Thus, the writer expresses the foreign opening degree of this region using the total import and export in one region. Qiu Rui (2010) also thinks that with the deepening of globalization, the economy of our country depends more on global economic market. The enhancement of total national economy level affects the foreign trade development more deeply.

Industrial development level is measured with the proportion of regional gross industrial production in regional GDP. Zhu Jia et al (2012) has ever proved that industrial agglomeration promotes regional economic development greatly, but the agglomeration in different regions at different extent exerts different effect on economic growth. Generally speaking, industrial development level promotes the economic development of this region to some extent.

Based on the above analysis, the writer set up the model as:

$$
\begin{aligned}
& G D P_{i t}=F\left(Q_{i t}, \operatorname{Inv}_{i t}, \operatorname{Con}_{i t}, \operatorname{Ind}_{i t}\right) \\
& =\theta_{1} \operatorname{Inv}_{i t}+\theta_{2} \operatorname{Con}_{i t}+\theta_{3} \text { Fore }_{i t}+\theta_{4} \operatorname{Ind}_{i t}+\beta_{i} Q_{i t} I\left(Q_{i t}, \gamma\right)+e_{i t}
\end{aligned}
$$

Hereinto, i=1, 2, 3,..., 9; 2000,2001,...,2014

All the original data used here come from Statistic Yearbook of Zhejiang Province, Financial Staistic Yearbook, Zhejiang Provincial Bureau of Statistics Website, Statistic Yearbook of nine regions of Zhejiang province, and national economy and social development statistic bulletin of nine regions from 2002 to 2016. Hereinto, all the indicators involving price factor adopt GDP 
deflator to get rid of the impact of price. Table 1 is the descriptive statistics of various indicators.

Table 1 Descriptive statistics of various indicators

\begin{tabular}{|c|c|c|c|c|}
\hline Variable name & Mean & $\begin{array}{c}\text { Standard } \\
\text { difference }\end{array}$ & Minimum & Maximum \\
\hline gdp & 10.171 & 0.696 & 8.848 & 11.452 \\
\hline q & 0.928 & 0.712 & 0.322 & 2.989 \\
\hline inv & 6.084 & 1.235 & 3.924 & 9.510 \\
\hline con & 0.958 & 0.458 & 0.332 & 2.556 \\
\hline fore & 5.056 & 1.742 & 1.327 & 8.485 \\
\hline ind & 0.276 & 0.402 & 0.007 & 2.015 \\
\hline
\end{tabular}

\section{Threshold value recognition}

According to description and analysis of threshold model in the previous texts, the number of threshold shall be determined firstly so as to determine the form of model. The following hypotheses of estimating equation shall be made as: no threshold, one threshold, and two thresholds. Consequently, we get $\mathrm{F}$ value and corresponding $\mathrm{P}$ value using Bootstrap sampling method, namely recognizing threshold value of financial agglomeration to regional economic growth effect. The test result is shown in Table 2 .

Table 2 Test result of threshold model hypothesis

\begin{tabular}{|c|c|c|c|c|c|}
\hline Threshold & & & \multicolumn{3}{|c|}{ Critical value } \\
\cline { 4 - 6 } model & F value & P value & $1 \%$ & $5 \%$ & $10 \%$ \\
\hline $\begin{array}{c}\text { Single } \\
\text { threshold }\end{array}$ & $7.867^{*}$ & 0.077 & 15.977 & 8.721 & 6.664 \\
\hline $\begin{array}{c}\text { Double } \\
\text { thresholds }\end{array}$ & $13.593^{*}$ & 0.080 & 36.371 & 19.308 & 12.502 \\
\hline $\begin{array}{c}\text { Triple } \\
\text { thresholds }\end{array}$ & 2.083 & 0.333 & 11.818 & 7.627 & 4.964 \\
\hline
\end{tabular}

(Note: $\mathrm{P}$ value and critical value are outcome of repeated sampling using Bootstrap sampling method for 300 times; “*” means significance below $10 \%$.)

According to Table 3, it is seen that single threshold and double thresholds are very significant when it is below $10 \%$ and the corresponding self-sampling $P$ value is 0.077 and 0.080 . However, the test result of triple thresholds is insignificant. Thus, we accept the hypothesis of two threshold value and select "double threshold model” for analysis. The estimated result is shown in Table 3.

Table 3 Estimates of double threshold

\begin{tabular}{|c|c|c|}
\hline Threshold value & $\begin{array}{c}\text { Estimated threshold } \\
\text { value }\end{array}$ & 95\% confidence interval \\
\hline$\gamma_{1}$ & 0.532 & {$[0.528,1.012]$} \\
\hline$\gamma_{2}$ & 0.982 & {$[0.570,2.839]$} \\
\hline
\end{tabular}

According to Table 4, it is seen that when the two threshold values of double threshold model are 0.532 and 0.982, the likelihood ratio approximates 0 . In addition, when the threshold value is in 95\% confidence interval, the likelihood value is smaller than critical value at $5 \%$ significant level.

After estimating threshold value, we estimate the parameters of model. Here, to eliminate the impact of heteroscedasticity on the result, conventional standard error method and robust standard error method are used as regression. The regression result of model is shown in Table 4. 
Table 4 Regression result of double threshold model

\begin{tabular}{|c|c|c|}
\hline & fe & fe_robust \\
\hline inv & $0.201^{* * *}(7.06)$ & $0.201^{* * *}(5.73)$ \\
\hline con & $0.539^{* * *}(5.63)$ & $0.539^{* *}(2.31)$ \\
\hline fore & $0.257^{* * *}(6.21)$ & $0.257^{* *}(3.24)$ \\
\hline ind & $0.405^{* *}(2.52)$ & $0.405^{*}(2.28)$ \\
\hline q_x_q1 & $-0.241(-1.41)$ & $-0.241(-1.03)$ \\
\hline q & $0.662^{* * *}(3.16)$ & $0.662(1.75)$ \\
\hline q_x_q3 & $-0.217^{* *}(-2.16)$ & $-0.217^{*}(-2.26)$ \\
\hline
\end{tabular}

\section{Conclusion}

The writer studies the threshold effect of financial agglomeration and regional economic growth based on panel data of nine regions in Zhejiang Province from 2002 to 2016. According to empirical study result, it is known that the relationship between financial agglomeration and regional economic growth was not merely simple linear function relationship, but mingled with "double threshold effect". That is, when the financial agglomeration level was low, the increasing of financial agglomeration can inhibit economic growth; when the financial agglomeration level was in the medium range, the increasing of financial agglomeration can promote economic growth effective; and when the financial agglomeration level was relatively high, the increasing of financial agglomeration has adverse effect on economic growth. In other words, compared with entity economy, the financial agglomeration inhibits the growth of regional entity economy no matter the financial agglomeration level is low or high and even if the channels of inhibiting the growth of entity economy is different.

\section{References}

[1] Arunkumar, N., Balaji, V.S., Ramesh, S., Natarajan, S., Likhita, V.R., Sundari, S. Automatic detection of epileptic seizures using independent component analysis algorithm (2012) IEEE-International Conference on Advances in Engineering, Science and Management, ICAESM-2012, art. No. 6215903, pp. 542-544.

[2] Yang Du, Yizheng Chen, Yiyang Zhuang, Chen Zhu, Fujian Tang, *Jie Huang. Probing Nanostrain via a Mechanically Designed Optical Fiber Interferometer. IEEE Photonics Technology Letters, 29(2017), pp. 1348-1351.

[3] Fernandes, S.L., Gurupur, V.P., Sunder, N.R., Arunkumar, N., Kadry, S.A novel nonintrusive decision support approach for heart rate measurement (2017) Pattern Recognition Letters. https://doi.org/10.1016/j.patrec.2017.07.002

[4] Arunkumar, N., Ramkumar, K., Venkatraman, V., Abdulhay, E., Fernandes, S. L., Kadry, S., \& Segal, S. (2017). Classification of focal and non-focal EEG using entropies. Pattern Recognition Letters, vol. 94, pp. 112-117

[5] Jennifer W. Chan, Yingyue Zhang, and Kathryn E. Uhrich, Amphiphilic Macromolecule Self-Assembled Monolayers Suppress Smooth Muscle Cell Proliferation, Bioconjugate Chemistry, 2015, 26(7), 1359-1369. 